\title{
Ghana's Ensure Mothers and Babies Regular Access to Care (EMBRACE) program: study protocol for a cluster randomized controlled trial
}

Kimiyo Kikuchi ${ }^{1}$, Evelyn Ansah², Sumiyo Okawa ${ }^{1}$, Akira Shibanuma ${ }^{1}$, Margaret Gyapong ${ }^{3}$, Seth Owusu-Agyei ${ }^{4}$, Abraham Oduro ${ }^{5}$, Gloria Quansah-Asare ${ }^{6}$, Abraham Hodgson ${ }^{2}$, Masamine Jimba ${ }^{1 *}$ and for the Ghana EMBRACE Implementation Research Project

\begin{abstract}
Background: The United Nations' Millennium Development Goals call for improving maternal and child health status. Their progress, however, has been minimal and uneven across countries. The continuum of care is a key to strengthening maternal, newborn, and child health. In this context, the Japanese government launched the Ghana Ensure Mothers and Babies Regular Access to Care (EMBRACE) Implementation Research Project in collaboration with the Ghanaian government. This study aims to evaluate the implementation process and effects of an intervention to increase the continuum of care for maternal, newborn, and child health status in Ghana.

Methods/Design: We will conduct a cluster randomized controlled trial using an effectiveness-implementation hybrid design in Dodowa, Kintampo, and Navrongo, Ghana. We will provide an intervention package to women living in randomly allocated intervention clusters. The study population is women of reproductive age between the ages of 15 and 49 years. The package includes: 1) use of a new continuum of care card, 2) continuum of care orientation for health workers, 3) 24-hour health facility retention of mothers and newborns after delivery, and 4) postnatal care by home visits. We will measure maternal, newborn, and child health outcomes for both intervention and implementation impacts. The intervention outcomes are continuum of care completion rate, rate of postnatal care within 48 hours, complication rate requiring mothers' and newborns' hospitalizations, and perinatal and neonatal mortality. The implementation outcomes are intervention coverage of the target population, intervention adoption and fidelity, implementation cost, and sustainability.

Discussion: In this trial, we will investigate how successful continuum of care can contribute to improving maternal, newborn, and child health outcomes. If successful, this model will then be implemented further in Ghana and other neighboring countries.
\end{abstract}

Trial registration: Current Controlled Trials ISRCTN90618993. Registered on 3 September 2014.

Keywords: Continuum of Care, Maternal, Newborn, and Child Health Outcomes, Ghana

\footnotetext{
*Correspondence: mjimba@m.u-tokyo.ac.jp

'Department of Community and Global Health, Graduate School of

Medicine, The University of Tokyo, 7-3-1 Hongo, Bunkyo-ku, Tokyo 113-0033,

Japan

Full list of author information is available at the end of the article
} 


\section{Background}

The United Nations' Millennium Development Goals (MDGs) 4 and 5 seek to improve maternal, newborn, and child health $(\mathrm{MNCH})$ status. Their progress, however, has been minimal [1-4] and uneven across countries, especially in Sub-Saharan Africa [5-8]. Unless drastic improvement is attained, most countries in Sub-Saharan Africa will not meet MDGs 4 and 5 [5].

In this context, global health agencies have advocated continuum of care $(\mathrm{CoC})$ as a new paradigm to overcome $\mathrm{MNCH}$ challenges $[9,10]$. The term $\mathrm{CoC}$ is well known in nursing for palliative $[11,12]$ and mental health care [13]. In the public health area, the concept of $\mathrm{CoC}$ has been used in HIV and AIDS care [12,14], linking HIV diagnosis, care initiation, therapy retention, and low viral load maintenance. However, for $\mathrm{MNCH}$, the definition of $\mathrm{CoC}$ has not yet been clearly established, although several attempts have been made [11,15-17]. So far, $\mathrm{CoC}$ has been explained by sequential time and space dimensions of $\mathrm{MNCH}$ activities. The time dimension lasts from pre-pregnancy for women through childhood. The space dimension links homes and communities through health facilities [17].

The $\mathrm{CoC}$ in $\mathrm{MNCH}$ provided a framework to improve health care for both women and their children by implementing integrated interventions $[11,18]$. Such intervention packages addressed different time stages in the $\mathrm{CoC}$ [19-21]. Additionally, single interventions were also efficacious for both women and their children [18]. Examples include newborn care preparedness by community-based approaches [22,23], use of insecticide-treated nets in pregnancy [24], and iron-folic acid supplementation [25,26]. Furthermore, financially focused strategies attempted to increase the demand for $\mathrm{MNCH}$ health services [27-30].

In the space dimension, the emphasized linkage is between the home and the first-level facility or the hospital. In most African countries, newborns are vulnerable to care delays and many newborn deaths occur at home [31]. Thus, interventions provided appropriate care at home, strengthened health system supports, and improved household and community practices and actions [32]. For both women and children, positive impacts were identified by training traditional birth attendants [33] and increasing emergency referral systems in the community [34]. Although these interventions improved $\mathrm{MNCH}$ outcomes, long-term sustainability remains a challenge [35], and it is unclear whether they were effective in 'real-world' settings. To make the best use of the available interventions, we need to know how they work in such settings [36].

Moreover, it is unclear whether health outcomes will be improved by filling the gaps in the CoC. A study estimated that neonatal mortality would be reduced by between 36 and $67 \%$ if all standard $\mathrm{MNCH}$ care packages (such as the antenatal care (ANC) package, skilled maternal and immediate newborn care package, and emergency obstetric care package) covered $90 \%$ of pregnant women $[37,38]$. However, evidence-based studies are scarce that demonstrate the effectiveness of improved $\mathrm{CoC}$ on health outcomes by addressing these gaps.

In 2010, the Japanese government presented new global health initiatives at the United Nations High-Level Plenary Meeting on MDGs [39]. The statement expressed a strategy to accelerate Japan's concerted efforts to help achieve health-related MDGs in developing countries, particularly for maternal and child health [40]. The initiative comprised a model program, the Ghana Ensure Mothers and Babies Regular Access to Care (EMBRACE) program. This program exploits a package of effective interventions to improve the health of mothers and children through the $\mathrm{CoC}$ approach. A special feature of the initiative was the Japanese government's clear intention to implement an 'evidence-based intervention' [41]. To extend the benefits of the Ghana EMBRACE initiative and evidence-based practices in health policy, the Ghana EMBRACE Implementation Research Project was launched in 2012 $[42,43]$. The project team comprised researchers from the University of Tokyo and the Ghana Health Service (GHS), including Dodowa, Kintampo, and Navrongo Health Research Centres (HRC). Based on formative research results, the project team developed interventions and will implement them in three Health and Demographic Surveillance System (HDSS) sites in Ghana.

Ghana is one of the countries that face a significant challenge to improving MNCH status [8,44-46]. Although it has made progress in different HDSS sites [47-52], according to the currently available evidence, Ghana is not on track to meet these MDGs [53]. In particular, $\mathrm{MNCH}$ status is poor in remote areas $[47,49]$, and care-seeking decisions are delayed for ill mothers and children [54,55]. Neonatal death is associated with maternal factors such as multiple gestations and inadequate birth spacing [56]. Additionally, mothers and children do not use all $\mathrm{MNCH}$ services continuously. In particular, mothers pay less attention to newborn care. In almost half of home deliveries, postnatal care (PNC) was not completed [45]. Approximately $40 \%$ of mothers who delivered at home reported that they received PNC for themselves, while only $16 \%$ reported receiving it for their newborns [57]. However, when mothers appropriately used the antenatal, delivery, and postnatal health services, the risk of neonatal death was reduced [56].

$\mathrm{MNCH}$ is often influenced by a complex interaction of economic, financial, social, cultural, and clinical factors. Ghana has multiple localities formed by the particular characteristics of each area. Poor infrastructure often limits access to emergency care [55]. In certain areas, a traditional illness, asram, could be one cause of careseeking delay for ill children [58]. The diversity of local 
characteristics implies the need for flexibility in health service provision $[59,60]$. This explains why implementation trials should be conducted in Ghana in different actual settings.

This study proposes specific objectives for the intervention and implementation phases. The intervention objective is to evaluate the impact of increased $\mathrm{CoC}$ completion on $\mathrm{MNCH}$ status in Ghana. The implementation objective is to evaluate intervention acceptability in different settings in Ghana.

In light of pragmatic situations in the intervention settings, we included the following as $\mathrm{CoC}$ components:

1. ANC delivered at least four times by $\mathrm{MNCH}$ health service providers at a health facility, in the community, or at home;

2. Delivery assisted by skilled birth attendants (SBAs); and

3. PNC delivered three times by $\mathrm{MNCH}$ health service providers at a health facility, in the community, or at home, within 48 hours, at seven days, and at six weeks postpartum.

\section{Methods/Design Study design}

We will conduct a cluster randomized controlled trial using an effectiveness-implementation hybrid design [36,61,62]. In this study design, we will assess the effects of both an intervention package and its implementation process. An effectiveness-implementation hybrid trial is an innovative design to shorten the time from intervention development through its implementation in a real-world setting $[63,64]$. This study design is categorized into three types. The type one design focuses on testing the intervention while observing or gathering information on the implementation. The type two design simultaneously tests the intervention and its implementation strategy. The type three design focuses more on testing implementation strategy while observing or gathering information on intervention outcomes [61]. In our study, we will adopt the type two hybrid design to determine both the impact of the intervention on $\mathrm{CoC}$ completion in $\mathrm{MNCH}$ and the acceptability of the implementation strategy by GHS.

This design will assess the effectiveness of intervention and implementation by using cluster randomized allocation to divide participants into intervention and control arms. For trial sustainability and scalability, we will consider site-specific contexts in designing interventions [59]. We will conduct the study in three stages: baseline, implementation, and follow-up phases. Acceptability of the trials will also be evaluated through monitoring ongoing trials.

\section{Study site}

We will conduct the study at three different sites in Ghana; Dodowa (Greater Accra region), Kintampo (Brong-Ahafo region), and Navrongo (Upper East region), where the GHS runs HDSS sites (Figure 1). These three sites were selected because highly reliable HDSS data are available. HDSS involves semi-annual recording of vital demographic events occurring among residents of all households in the HDSS area: pregnancies, births, deaths, and migration. Other data are also collected and updated regularly, such as economic status, morbidity, and vaccination.

At the Dodowa HDSS site, the population was approximately 115,000 in 2011. It is located about 40 kilometers away from Accra [65]. Consequently, pregnant women from this site often prefer to deliver at health facilities in Accra. Service deliveries are challenging since the site land size covers about $40.5 \%$ of the Greater Accra region. Most of the communities are scattered, containing small populations $[65,66]$.

At the Kintampo HDSS site, the surveillance population was approximately 200,000 in 2011 . This site is a multiethnic area and farming is the most important economic activity. Apart from the central area, most villages are not supplied with electricity and are reached by dirt roads. Access to health facilities is a challenge in Kintampo [65,67], and a home delivery is often the first choice [68].

At the Navrongo HDSS site, the surveillance population was approximately 153,000 in 2011 [65,69]. Navrongo is the first area where the Community-based Health Planning and Services (CHPS) program was launched in Ghana. In that context, Community Health Officers (CHOs) have contributed to improving health status in communities. Among the three HDSS sites, only Navrongo is on track to achieve MDG 5 [48].

As the $\mathrm{MNCH}$ services are national priority areas in Ghana, various $\mathrm{MNCH}$ interventions have been implemented in the three HDSS sites. Representative interventions include Mobile Technology for Community Health (MoTeCH, between 2009 and 2012) [70], Quality of prenatal and maternal care (Qualmat, between 2009 and 2014) [71], and Ghana Essential Health Interventions Programme (GEHIP, between 2009 and 2014) [72] in Navrongo; early neonatal vitamin A supplementation in improving child survival (Neovita, between 2010 and 2012) [73], and Newborn home intervention study project (Newhints, between 2008 and 2010) [74] in Kintampo; and the conditional cash transfer project (GLST, between 2009 and 2014) [75] and Neonatal Quality Improvement Programme (NQIP, between 2011 and 2012) in Dodowa.

\section{Randomization and allocation}

Each HDSS site contains two districts, and the districts comprise multiple sub-districts, which are the minimum health administration units in Ghana. We defined the sub-district as a cluster unit. Only Jema and Dumso (which used to be a part of Jema) sub-districts in the Kintampo site were combined to form a cluster. In the 


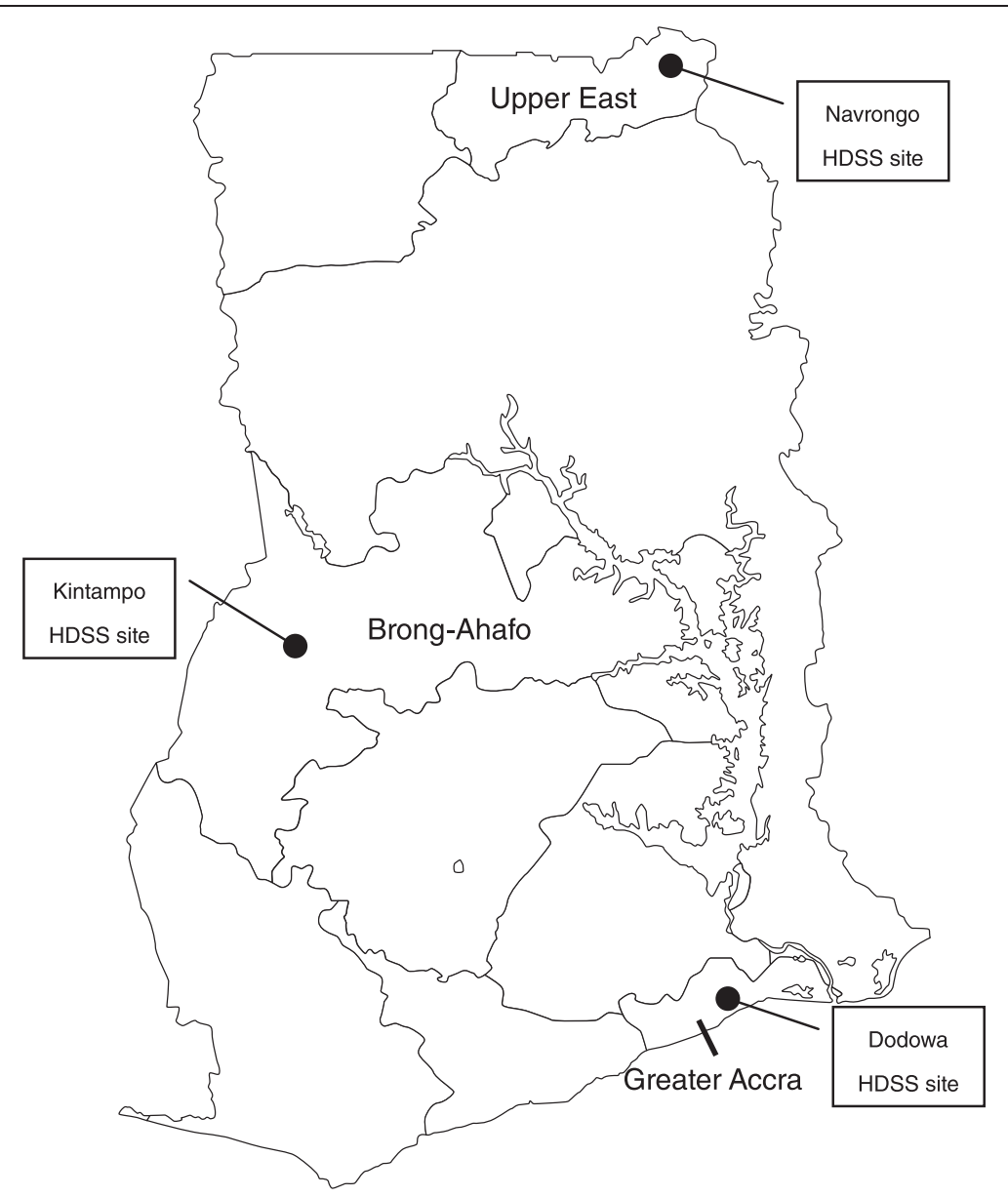

Figure 1 Map of health and demographic surveillance system sites. Ghana has health and demographic surveillance system (HDSS) sites in Dodowa, Kintampo, and Navrongo. The HDSS areas involve highly reliable semi-annual recording of vital demographic events occurring in residents of all households.

three HDSS sites, a total of 36 sub-districts were included. Of them, we excluded four sub-districts of Navrongo where another $\mathrm{MNCH}$ project is planned. In total, we chose 32 clusters for our study targets (Figure 2): eight clusters in Dodowa, 12 clusters in Kintampo, and 12 clusters in Navrongo. We allocated half of the subdistricts to the intervention arm and the other half to the control arm. At least one hospital was allocated to the intervention arm in each site, since the majority of facility deliveries are done in hospitals (Table 1). Due to the nature of the intervention, masking was not feasible.

We matched the clusters within the site before randomization in the following aspects: population, number of deliveries in each cluster, and number of midwives available. Cluster randomization was preferred over individuallevel randomization to minimize contamination, and also for the pragmatic purposes of a future scale-up of the intervention. The clusters were randomized by a data analyst, who was not a primary member of the study team, using computer-generated random sequences.

\section{Study population and selection criteria}

Our study population is women of reproductive age between the ages of 15 and 49 years who live in the areas covered by the Dodowa, Kintampo, and Navrongo HDSS sites. Participants will be women who have given birth in the study area between 1 September 2012 and 30 June 2014 for the baseline period, and between 1 October 2014 and 30 September 2015 for the trial period. Exclusion criteria for the trials are women who refuse to participate in the intervention. We expect only a few women will meet it as the interventions will be implemented within the current service delivery system. In other words, they will receive the interventions unless they refuse national standard $\mathrm{MNCH}$ services. Exclusion criteria for the impact evaluations are those who decline to be interviewed or who have migrated out of the HDSS sites. We will also involve health service providers in the intervention to provide $\mathrm{CoC}$ services and to educate participant women in the $\mathrm{CoC}$ concept. 


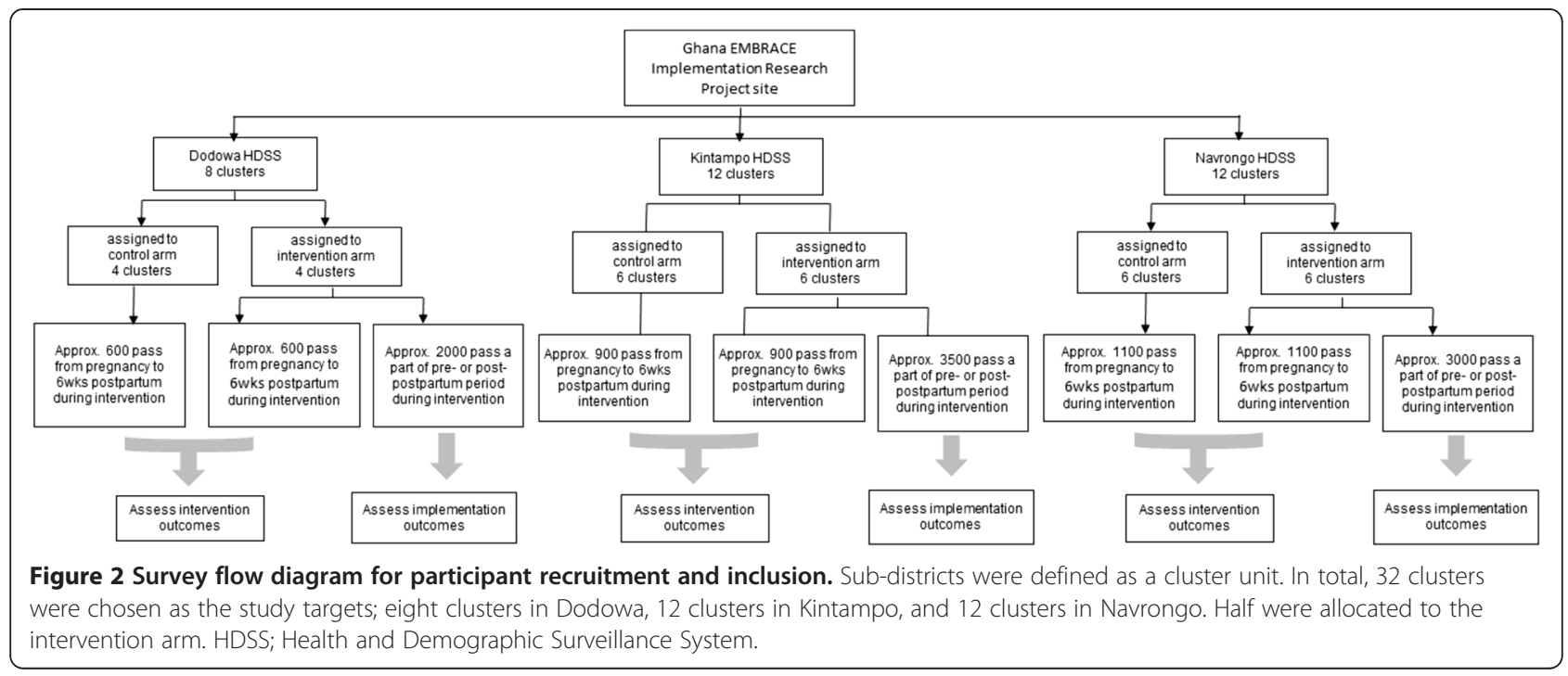

\section{Recruitment and consent of participants}

We will deliver the interventions to all eligible women in the intervention arm. $\mathrm{MNCH}$ service providers will approach them when receiving $\mathrm{MNCH}$ care at health facilities or home. The expected recruitment period will be from August to September 2014 for the baseline survey, from October 2014 to September 2015 for the intervention, and from October to November 2015 for the follow-up survey. We will request informed consent form signatures from the survey participants. Additionally, we will obtain participants' oral consent at their intervention entry point. Their participation will be voluntary and they are free to join or withdraw at any time.

\section{The Ghana EMBRACE interventions}

\section{Development of an intervention package}

We developed a package of interventions based on a formative research survey conducted in three different HDSS sites (Table 2). In the survey, we identified the gaps, barriers, and promoters of service reception in continuous $\mathrm{MNCH}$ care. To address the actual $\mathrm{MNCH}$

Table 1 Number of facilities (As of June 2014)

\begin{tabular}{llrrrr}
\hline HDSS site & Arm & Hospital & $\begin{array}{c}\text { Health } \\
\text { center }\end{array}$ & $\begin{array}{c}\text { Functional } \\
\text { CHPS zone }\end{array}$ & Private \\
\hline Dodowa & Intervention & 1 & 1 & 13 & 5 \\
& Control & 0 & 3 & 21 & 4 \\
\multirow{2}{*}{ Kintampo } & Intervention & 1 & 2 & 19 & 5 \\
& Control & 1 & 4 & 22 & 0 \\
\multirow{2}{*}{ Navrongo } & Intervention & 1 & 3 & 18 & 4 \\
& Control & 0 & 3 & 18 & 0 \\
\hline
\end{tabular}

CHPS, Community-based Health Planning and Services; HDSS, Health and Demographic Surveillance System. conditions in Ghana and site-specific cultural differences in delivery location, geographic conditions, and health system capacity, we developed the following four interventions: the utilization of the $\mathrm{CoC}$ card (A-1), $\mathrm{CoC}$ orientation (A-2), 24-hour health facility retention of mothers and newborns after delivery (B-1), and PNC by home visits (B-2). We will implement $\mathrm{A}-1, \mathrm{~A}-2$, and B-2 interventions in all three HDSS sites. However, B-1 will be implemented only in Dodowa and Navrongo because the number of midwives is not enough in Kintampo.

In the control arm, Ghanaian government will provide conventional $\mathrm{MNCH}$ services. In most of the primary level health facilities, basic $\mathrm{MNCH}$ services will be provided. The delivery and laboratory services are provided mainly in the secondary and tertiary level health facilities. ANC services include general check-ups for mothers, urine tests, hemoglobin tests, prevention of mother-to-child transmission (PMTCT) of HIV, nutritional support, tetanus toxoid immunization, and health education for birth preparedness and maternal complications. Delivery services include skilled delivery, facility referral, and emergency obstetric care. PNC services include general check-ups for mothers and children, body weights, infant vaccinations, hemoglobin tests, nutritional support, health education for breastfeeding and child care, and family planning counselling. Home visit check-ups are also provided, however, home visit PNC within 48 hours is poorly adopted.

\section{A-1 Utilization of the continuum of care card}

In addition to the maternal health record book, health service providers will provide the $\mathrm{MNCH} \mathrm{CoC}$ card to all women who receive ANC, delivery, and PNC assisted by SBAs (Table 3). When these women receive follow-up services, health service providers place a sticker on the 
Table 2 Interventions of Ghana EMBRACE implementation research project

\begin{tabular}{|c|c|c|}
\hline Interventions & Implementations & Implementers \\
\hline (A-1) Utilization of CoC card & $\begin{array}{l}\text { Encourage mothers to receive four } \\
\text { ANC visits, facility delivery, PNC } \\
\text { within } 48 \text { hours, at } 7 \text { days, and at } 6 \\
\text { weeks }\end{array}$ & $\begin{array}{l}\text { Health service providers who } \\
\text { routinely provide } \mathrm{MNCH} \text { at health } \\
\text { facility, community, or home: doctor, } \\
\text { midwife, nurse, } \mathrm{CHO} \text {, and } \mathrm{CHN}\end{array}$ \\
\hline \multirow[t]{2}{*}{$\begin{array}{l}\text { (A-2) Orientation of health } \\
\text { service providers }\end{array}$} & $\begin{array}{l}\text { Reorient supervisors in } \\
\text { understanding CoC }\end{array}$ & $\begin{array}{l}\text { Master trainers from GHS/EMBRACE } \\
\text { researchers }\end{array}$ \\
\hline & $\begin{array}{l}\text { Reorient health service providers in } \\
\text { understanding CoC }\end{array}$ & $\begin{array}{l}\text { Trainer from DHMT/SDHMT who } \\
\text { attended the training of trainers }\end{array}$ \\
\hline
\end{tabular}

(B-1) 24-hour retention of mother and their newborns after delivery

(B-2) PNC by home visits
Provide PNC within 48 hours by retaining mothers with newborns for at least 24-hours postpartum

Provide PNC within 48 hours by visiting the home of mothers
Health service providers working at district hospital, health center, CHPS compound, or private clinic with midwife

Health service providers working at health center or CHPS compound

\author{
Targets \\ Women in prepartum/postpartum \\ period who come to the health \\ facility located in the intervention \\ arm for ANC, delivery, and PNC \\ Regional Health Administrations, \\ DHMT, and SDHMT \\ Primary maternal and child health \\ service providers of the health \\ facilities in the intervention sites: \\ midwife, $\mathrm{CHO}, \mathrm{CHN}$, doctor, nurse, \\ and health assistant.
}

Mothers who delivered at a relevant health facility and their newborns

Mothers who gave birth but did not stay for 24 hours after delivery

Mothers who delivered at home and their newborns

ANC, antenatal care; CHN, Community Health Nurse; CHO, Community Health Officer; CHPS, Community-based Health Planning and Service; CoC, Continuum of Care; DHMT, District Health Management Team; SDHMT, Sub-District Health Management Team; GHS, Ghana Health Service; MNCH, maternal, newborn, and child health; PNC, postnatal care.

card to show that they complied with the CoC; this includes four ANC visits, delivery assisted by SBAs, and three PNC visits (within 48 hours, at seven days, and at six weeks after delivery). Additionally, health service providers record on the $\mathrm{CoC}$ card: provision of essential services and health education; blood tests for assessing

Table 3 Maternal, newborn, and child health continuum of care card contents

\begin{tabular}{ll}
\hline Contents & Main components \\
\hline CoC services & Four ANC visits \\
& Delivery with skilled birth attendant \\
& Three PNC visits \\
Essential services & Hemoglobin test \\
& Malaria drug (IPT) \\
& Tetanus toxoid immunization \\
& Blood group and Rhesus factor \\
Items for delivery and baby & Caregiver \\
Health education & Transportation for delivery \\
& Call a health service provider after delivery \\
& Early initiate and exclusive breast feeding \\
Family planning counselling & Identification of danger signs during pregnancy \\
Identification of danger signs at delivery and \\
after delivery for mother \\
Identification of danger signs at birth and after \\
birth for newborn
\end{tabular}

ANC, antenatal care; CoC, Continuum of Care; IPT, Intermittent Preventive Treatment; PNC, postnatal care. hemoglobin, blood group, and Rhesus factor; intermittent preventive treatment (IPT) for malaria; tetanus toxoid immunization; early initiating and exclusive breastfeeding; family planning; preparing items for delivery and baby; arrangement of transportation for delivery, caregiver, and calling health service providers after delivery; and the presence of danger signs during pregnancy and after delivery for mother and newborn.

\section{A-2 Continuum of care orientation}

We will implement the $\mathrm{MNCH} \mathrm{CoC}$ orientation in two stages. First, we will complete the training of trainers for supervisors of District Health Management Teams (DHMT) and Sub-District Health Management Teams (SDHMT). The trained supervisors will then conduct orientations for health service providers at hospitals, health centers, CHPS, and private clinics. We will focus mainly on introducing $\mathrm{MNCH} \mathrm{CoC}$ concepts, their importance, the extent to which the $\mathrm{MNCH} \mathrm{CoC}$ card could be used, and the protocols of other interventions.

\section{B-1 24-hour health facility retention of mothers and newborns after delivery}

In this intervention, we will encourage mothers to stay with their newborns at health facilities after delivery for at least 24 hours for PNC. This intervention targets only the health facilities where SBAs provide delivery services. In this intervention, mothers and newborns will receive necessary care in a health facility during the 24 hours after delivery. During their stay, we will provide them nutritious drink supplements (for example, Milo (Nestlé S.A., Vevey, Switzerland)). After ensuring all of the necessary health 
check points, health service providers will discharge them if neither mothers nor babies show any danger signs.

\section{B-2 Postnatal care by home visits}

We will encourage $\mathrm{CHOs}$ to visit mothers and newborns for PNC within 48 hours after delivery. This intervention is composed of two steps. The first step is delivery notification; when home delivery occurs, community health volunteers, community key informants, or traditional birth attendants will inform the $\mathrm{CHOs}$ that the labor or delivery occurred in the community. The second step is PNC by home visits; $\mathrm{CHOs}$ visit mothers and newborns for PNC within 48 hours postpartum.

\section{Procurement}

Where necessary, we will provide beds for postpartum rest, rechargeable lamps or solar lanterns, torchlights for all B-1 intervention facilities, and motorbikes for the B-2 intervention facilities. We will also provide care materials for both eligible categories of health facility such as blood pressure apparatus for mothers and children, stethoscopes, thermometers, and pen lights.

\section{Supportive activities}

We will organize three types of supportive activities before the start of the intervention to implement the interventions smoothly into the communities. They include stakeholder meetings, community leader meetings, and community durbars. We will organize the stakeholder meetings in both the intervention and control arms. To avoid contamination of the intervention effect, the community leader meetings and community durbars will be held only in the intervention arm.

\section{Monitoring}

In each $\mathrm{HRC}$, we will form an intervention monitoring team. This team is responsible for monthly monitoring of all implementation activities of health service providers and for supervision of the DHMT/SDHMT supervisors. The EMBRACE researchers will meet monthly to discuss the issues raised during the monitoring and provide feedback to the monitoring team and the DHMT/SDHMT supervisors.

\section{Data collection}

We will assess the impact of the intervention by interview survey using a semi-structured questionnaire and HDSS data. We will also assess the acceptability of the implementation process by monthly monitoring and supervision, and by conducting a survey of eligible women at the HDSS sites. Data managers of the HRCs will review all of the collected data for accuracy and completion. Data will be entered into computers twice, using Visual FoxPro software (Microsoft, Washington,
United States). Verification checks will be done to correct any discrepancies in records.

\section{Evaluation of intervention efficacy}

We will assess the intervention efficacy using both survey and HDSS data, as described below:

1. By survey: to assess the intervention impact, we will conduct the interview survey at baseline and follow-up periods for sampled eligible women. For each survey, we will recruit approximately 500 women from each HDSS site (in total, 1,500 women for each survey). We will select them through the HRC pregnancy registers using the following two steps: (a) creating geographical units (GU) in proportion with the population size of the cluster and selecting 50 GUs from each HDSS site, and (b) selecting 10 eligible women from each GU. We will select the GUs and women using computer-generated random numbers. The questionnaire items are: sociodemographic and socioeconomic characteristics; $\mathrm{MNCH}$ services uptake; health complications during pregnancy, at delivery, and during postnatal period; pregnancy outcomes; and care-seeking behaviors. To control for the effects of health care provider and health facility characteristics on the study outcome, we will conduct health care provider assessment and health facility assessment at the baseline survey for both intervention and control arms. The data we will collect includes information on service provision, knowledge of $\mathrm{CoC}$, supervision, community support, job satisfaction at health care provider level, infrastructure, human resources, $\mathrm{MNCH}$ service availability, annual statistics of key $\mathrm{MNCH}$ indicators, current practice of retaining mother and newborn for the first 24 hours, and provision of PNC by home visit within 48 hours postpartum. Prior to the survey, we will complete training for research assistants at each site. The EMBRACE researchers will develop the survey questionnaire through several workshops based on the formative research questionnaire. We will develop the questionnaire in English and the research assistants will interpret it orally when they conduct interviews with participants. As the local languages are not always written, we will not use translated questionnaires. The trained research assistants will pre-test the questionnaire with 20 eligible women and the researchers will revise it accordingly.

2. By HDSS data: we will also obtain the HDSS data to assess the perinatal mortality rate (PMR) and the neonatal mortality rate (NMR) of HDSS sites. We will officially request that HRCs share the relevant 
data with the Ghana EMBRACE Implementation Research Project.

\section{Evaluation of implementation process acceptability}

We will assess the acceptability of the implementation process by monitoring, supervision, key informant interviews, and surveys, as described below:

1. By monitoring: to assess intervention adoption, we will collect data about service provision through monthly monitoring. Data sources will be the health facility register book and District Health Information Management System (DHIMS) reports. The EMBRACE researchers developed the monitoring items through several workshops.

2. By supervision: to assess intervention fidelity, we will collect monthly supervision data to check the consistency of intervention implementation.

3. By key informant interviews: we will conduct key informant interviews among DHMT and SDHMT supervisors, health service providers, and mothers in the intervention arm.

4. By survey: we will ask about health education and essential services provided to the women in the baseline and follow-up surveys. We will ask women in the intervention arm about service preferences through the follow-up survey.

\section{Outcome measures}

\section{Intervention outcome measures}

The primary outcome of the intervention is $\mathrm{CoC}$ completion rate of mothers and their children (Figure 3 and Table 4). The secondary outcomes include the PNC rate within 48 hours, the complication rate requiring mothers' and newborns' hospitalizations, and the PMR and NMR. The PMR is defined as fetal deaths during any period of pregnancy and newborn deaths within seven completed days after birth. The NMR includes early neonatal deaths occurring during the first seven days of life and late neonatal deaths occurring after seven days but before 28 completed days of life [76,77].

\section{Implementation outcome measures}

The implementation outcomes will be measured by five outcomes: 1) intervention coverage of target population, 2) adoption and 3) fidelity in CoC card utilization or PNC within 48 hours by mothers' retention at health facility or by home visit, 4) implementation cost, and 5) sustainability $[64,78,79]$.

\section{Sample size}

We made a calculation for two types of sample size to measure different outcomes. At first, to measure the
CoC completion rate, we used the interview survey and calculated a total of 1,500 women for the sample size. Second, to measure the NMR, we used a HDSS data and calculated a total of 15,000 women for the sample size.

To calculate the sample size of the $\mathrm{CoC}$ completion rate, we used the data of the formative research collected in the previous year in the same sites. According to the data, the $\mathrm{CoC}$ completion rate was $8.0 \%$. The coverage of four ANC visits, delivery attended by SBAs, PNC within 48 hours postpartum, and PNC at two weeks were $86.6 \%, 75.8 \%, 13.0 \%$, and $60.0 \%$, respectively. The lowest coverage was identified at PNC within 48 hours postpartum, and if this coverage increases to more than that of PNC at two weeks, the CoC completion rate would also increase to $60.0 \%$. For that, we estimated that coverage of four ANC visits would be improved from 86.6 to $95.0 \%$, and calculated the sample size using an intraclass correlation coefficient (ICC) of 0.02675 determined in the formative research. The ICC was estimated by considering the differences in the sizes of clusters using multilevel regression with a random intercept at the cluster level. The confidence interval (CI) was $95 \%$, and power was $80 \%$. Adding $10 \%$ for potential attrition, the total sample size was calculated at 1,500 for each baseline and follow-up survey period. Therefore, we estimated approximately a 500-person sample size for each HDSS site (1,500 in total).

In addition to the interview survey, we will also use the HDSS data to evaluate the effect of the interventions on perinatal and neonatal mortality. For the baseline and follow-up survey periods, HDSS data will capture 15,000 pregnancy cases, respectively. The sample size was estimated according to the following assumptions: a $25 \%$ reduction of PMR (from 31 to 23 per 1,000 pregnancies), with $95 \% \mathrm{CI}, 80 \%$ power, 32 clusters, and an ICC of 0.0007256 . The ICC was based on previous $\mathrm{MNCH}$ research conducted in the study area [74] and the sample size was calculated at 13,548 . In addition, we added $10 \%$ for potential attrition.

\section{Statistical analysis \\ Continuum of care completion and morbidity}

We will conduct baseline and follow-up interview surveys to assess changes in each outcome in both the intervention and control arms. To minimize overestimation of intervention impact, we will estimate all intervention impact outcomes with an intention-to-treat analysis. In this analysis, individuals' outcome data are analyzed according to the allocated arm regardless of the place where they received care. Also, all eligible individuals are included in the analysis regardless of whether they provided outcome data [80]. 


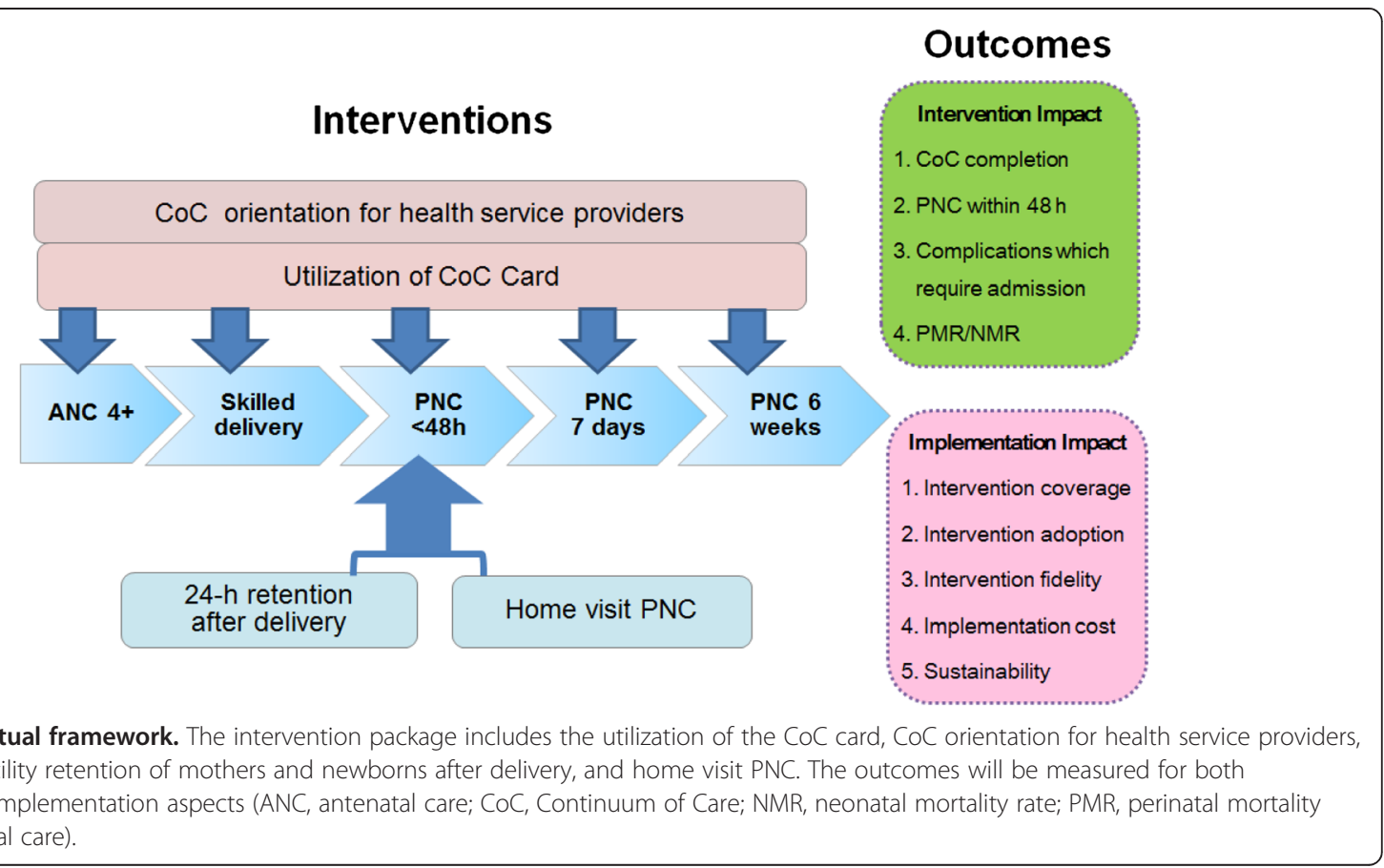

We will conduct a descriptive analysis to assess the distribution of various factors related to the mothers and newborns under study. To evaluate the average effectiveness of interventions on $\mathrm{CoC}$ completion, we will apply the generalized estimating equations model with both continuous and binary outcomes [80]. This model uses data on all mothers, including those with incomplete data, over the period from 16 weeks of pregnancy to six weeks postpartum. Adjustment factors include basic demographic characteristics, socioeconomic characteristics, and facility characteristics. The data analysis will mostly be conducted using Stata version 13 (StataCorp LP, College Station, Texas, United States).

\section{Mortality rate}

PMR and NMR will be calculated based on the number of live births and perinatal deaths or neonatal deaths as a total of the three HDSS sites, as well as at each HDSS site. These outcomes will be compared before and after interventions.

\section{Adoption of postnatal care within 48 hours and continuum of care card utilization}

Implementation impact will be evaluated by means of descriptive statistical analysis. The qualitative data will be coded and categorized.

\section{Ethical considerations}

\section{Approval}

Ethical approval was obtained from the Ethics Review Committee of the GHS (reference: GHS-ERC: 13/03/14), the Institutional Review Boards of Dodowa HRC (reference: FGS-DHRC: 280214), Kintampo HRC (reference: 2014-11), and Navrongo HRC (reference: NHRCIRB137) in Ghana, and from the Research Ethics Committee of the University of Tokyo in Japan (reference serial number: 10513).

\section{Individual and community consent}

Informed consent will be obtained from all survey participants before their inclusion in the study. We will record the consent through a signature or thumbprint. Participants will be withdrawn from the study if they experience a serious or intolerable adverse event, develop or disclose symptoms or conditions listed in the exclusion criteria, or require early discontinuation for any other reason. This will not affect their normal service delivery at any of the health facilities.

Permission for conducting the intervention study will be sought from the local health authorities and community leaders before initiating the study.

\section{Benefits and risks}

This intervention package is not invasive. Thus, the participants will not be exposed to marked risks. By participating in this study, they benefit by improved CoC knowledge and care of mothers and newborns. The $\mathrm{MNCH}$ service providers will receive a training session about $\mathrm{CoC}$ and the procedures to be performed. Health facilities of the intervention arm will receive a set of PNC services, motorbikes, or rest beds if they are not available ones. We will introduce the intervention to the 
Table 4 Outcomes of Ghana EMBRACE implementation research project

\begin{tabular}{|c|c|c|}
\hline Study objective & Outcome variables & \\
\hline \multicolumn{3}{|l|}{ Intervention outcomes } \\
\hline \multirow[t]{2}{*}{$\begin{array}{l}\text { To examine the effect } \\
\text { of the EMBRACE } \\
\text { interventions on CoC } \\
\text { completion }\end{array}$} & 1) CoC completion rate & $\begin{array}{l}\text { Rate of mothers and their newborns } \\
\text { who completed CoC: ANC } 4 \text { visits, } \\
\text { delivery assisted by SBAs, PNC } 3 \\
\text { times (within } 48 \text { hours, at } 7 \text { days, at } \\
6 \text { weeks postpartum) }\end{array}$ \\
\hline & $\begin{array}{l}\text { 2) Rate of PNC within } \\
48 \text { hours }\end{array}$ & $\begin{array}{l}\text { PNC rate of mothers and newborns } \\
\text { within } 48 \text { hours postpartum by } 24 \\
\text { hours retention at health facility or } \\
\text { home visit }\end{array}$ \\
\hline \multirow[t]{2}{*}{$\begin{array}{l}\text { To evaluate the impact } \\
\text { of the interventions on } \\
\text { MNCH status }\end{array}$} & $\begin{array}{l}\text { 3) Complication rate of } \\
\text { mothers and newborns }\end{array}$ & $\begin{array}{l}\text { Complication rates which require } \\
\text { mothers' and newborns' } \\
\text { hospitalizations for more than } 24 \\
\text { hours }\end{array}$ \\
\hline & 4) $P M R$ and $N M R$ & $\begin{array}{l}\text { PMR: fetal deaths during any period } \\
\text { of pregnancy and newborn death } \\
\text { within } 7 \text { completed days after birth. } \\
\text { Early NMR: neonatal deaths occurring } \\
\text { during the first } 7 \text { days of life. } \\
\text { Late NMR: neonatal deaths occurring } \\
\text { after } 7 \text { days but before } 28 \text { completed } \\
\text { days of life }\end{array}$ \\
\hline
\end{tabular}

\section{Implementation outcomes}

\begin{tabular}{|c|c|c|}
\hline \multirow[t]{4}{*}{$\begin{array}{l}\text { To evaluate the } \\
\text { acceptability of the } \\
\text { interventions in } \\
\text { different settings in } \\
\text { Ghana }\end{array}$} & $\begin{array}{l}\text { 1) Intervention } \\
\text { coverage }\end{array}$ & $\begin{array}{l}\text { Percentage of women covered by } \\
\text { intervention }\end{array}$ \\
\hline & 3) Fidelity & $\begin{array}{l}\text { Adherence to the protocol and } \\
\text { quality of intervention delivery }\end{array}$ \\
\hline & $\begin{array}{l}\text { 4) Implementation } \\
\text { cost }\end{array}$ & $\begin{array}{l}\text { Direct measures of implementation } \\
\text { cost and additional expense of } \\
\text { implementation costs }\end{array}$ \\
\hline & 5) Sustainability & $\begin{array}{l}\text { Institutionalization of interventions } \\
\text { or practice } \\
\text { Passage, cycle or routine, and niche } \\
\text { saturation }\end{array}$ \\
\hline
\end{tabular}

Methods Data source

Quantitative analysis Interview using semistructured questionnaire

Monitoring data

Quantitative analysis

Interview using semistructured questionnaire

Monitoring data

Quantitative analysis

Interview using semistructured questionnaire

Quantitative analysis

HDSS data

Interview using semistructured questionnaire
Quantitative analysis

Quantitative analysis

Qualitative summary of key informant interviews

Quantitative analysis

Qualitative summary of key informant interviews Monthly meeting of project coordinators

Quantitative summary

Discussions incorporating results of intervention research with project supervisors
Interview using semistructured questionnaire

Monitoring data

Monitoring data

Supervision data

Notes from key informant interview

Monitoring data

Supervision data

Notes from key informant interview

Discussion notes of coordinators' meetings

Costing format

Discussion notes of project meetings including district health officer

Notes from key informant interview with health workers and district health officers

Qualitative summary of

key informant interviews

ANC, antenatal care; CoC, Continuum of Care; HDSS, Health and Demographic Surveillance System; MNCH, maternal, newborn, and child health; NMR, neonatal mortality rate; PMR, perinatal mortality rate; PNC, postnatal care; SBA, skilled birth attendant.

control arm participants immediately when a positive impact is identified. We will encourage referral to a health facility if we identify minor, acute, or chronic illness in mothers or newborns in either the intervention or control arms.

\section{Confidentiality of information}

All information obtained through this study will be confidential. Access to information will be limited to research assistants for conducting interviews and data entry management staff. Study records will be 
identified only by means of study identification numbers.

\section{Dissemination of trial findings}

The results of the study will be presented first to community members and their leaders in HDSS sites. In addition, policy briefs will be developed in collaboration with the Policy, Planning, Monitoring, and Evaluation Division of the GHS and submitted to the Office of the DirectorGeneral of GHS and the Family Health Division. Presentations will be made at the GHS Directors meeting, the Senior Managers Meeting, and international conferences.

Trial findings will also be disseminated in scientific meetings and papers on the intervention impact on improving $\mathrm{CoC}$, the impact of increased $\mathrm{CoC}$ on $\mathrm{MNCH}$ status, the acceptability of the interventions, and the strategies for Japan's international health policy for $\mathrm{MNCH}$.

\section{Discussion}

This paper describes the protocol for a cluster randomized controlled trial to evaluate the impact of increased $\mathrm{CoC}$ completion on $\mathrm{MNCH}$ status and the acceptability of the interventions in different settings in Ghana. The interventions will be implemented in close consultation with local health administrative offices that have direct responsibility for supervising health facilities. Ongoing feedback will be provided through routine supervision. Additionally, we developed the study materials in close cooperation with policymakers in Ghana.

The study has limitations. First, the short intervention period may limit the power to fully measure the intervention and implementation impacts. The period is short because we needed repeated discussions to develop intervention packages aimed at a sustainable and scalable intervention design in a real-world setting. The project takes into account the next scale-up phase which will likely last years, during which the impacts could be measured more accurately. To reduce this limitation, we adopted the effectiveness-implementation hybrid trial that is an advantageous study design for time efficiency that enables rapid scaling of the intervention up to the national $\mathrm{MNCH}$ service standard. Second, our intervention impacts could be affected by previous or current projects implemented at the HDSS sites. To minimize it, we will control the potential effect for data analysis and carefully discuss the study findings.

\section{Trial status}

The trial was registered in the International Standard Randomized Controlled Trial Number Register on 3 September 2014 (ISRCTN90618993). Recruitment for intervention commences in October 2014 and will continue until September 2015.

\begin{abstract}
Abbreviations
ANC: Antenatal care; CHO: Community Health Officer; CHPS: Communitybased Health Planning and Services; Cl: Confidence interval; CoC: Continuum of Care; EMBRACE: Ensure Mothers and Babies Regular Access to Care; GHS: Ghana Health Service; GU: Geographical Unit; HDSS: Health and Demographic Surveillance System; HRC: Health Research Centre;

ICC: Intraclass correlation coefficient; MDGs: Millennium Development Goals; MNCH: Maternal, newborn and child health; NMR: Neonatal mortality rate; PMR: Perinatal mortality rate; PMTCT: Prevention of mother-to-child transmission; PNC: Postnatal care; SBA: Skilled birth attendant.
\end{abstract}

\section{Competing interests}

The authors declare that they have no competing interests.

\section{Authors' contributions}

KK and EA prepared the first draft of the manuscript. SO and MJ provided critical comments on the revision of the manuscript. AS provided input on the statistical analysis. MG, SOA, AO, GQA, and AH provided critical comments on the final manuscript. All listed co-authors and the Ghana EMBRACE Implementation Research Project Team contributed to the original trial design. All listed authors have read and approved the final manuscript.

\section{Acknowledgements}

The authors thank the Ministry of Foreign Affairs in Japan and the Ministry of Health in Ghana. Special thanks to the many advisers and implementation members.

The Ghana EMBRACE Implementation Research Project is conducted by the Government of the Republic of Ghana, Japan International Cooperation Agency (JICA) Human Development Department, and JICA Research Institute. The content is solely the responsibility of the authors and does not necessarily represent the official views of JICA Human Development Department and JICA Research Institute.

Ghana EMBRACE Implementation Research Project Team (in addition to named authors): Ebenezer Appiah-Denkyira, Yoshiharu Yoneyama, Junko Yasuoka, Keiko Nanishi, Yusuke Kamiya, Akiko Hagiwara, Sakiko Shiratori, Sheila Addei, Doris Sarpong, Charlotte Tawiah, Yeetey Enuameh, Kwame Adjei, Cornelius Debpuur, and Francis Yeji.

\section{Author details}

'Department of Community and Global Health, Graduate School of Medicine, The University of Tokyo, 7-3-1 Hongo, Bunkyo-ku, Tokyo 113-0033, Japan. ${ }^{2}$ Research and Development Division, Ghana Health Service, MB 190 Accra, Ghana. ${ }^{3}$ Dodowa Health Research Centre, PO Box DD1, Dodowa, Greater Accra, Ghana. ${ }^{4}$ Kintampo Health Research Centre, PO Box 200, Kintampo, Brong-Ahafo, Ghana. ${ }^{5}$ Navrongo Health Research Centre, PO Box 114, Navrongo, Upper East, Ghana. ${ }^{6}$ Ghana Health Service, Accra, Ghana.

Received: 9 September 2014 Accepted: 22 December 2014

Published online: 27 January 2015

\section{References}

1. Ngom P, Akweongo P, Adongo P, Bawah AA, Binka F. Maternal mortality among the Kassena-Nankana of northern Ghana. Stud Fam Plann. 1999;30:142-7.

2. United Nations International Children's Fund (UNICEF). Levels and trends in child mortality 2013. New York: UNICEF; 2013.

3. World Health Organization (WHO). Revised 1990 estimate of maternal mortality: a new approach by WHO and UNICEF. Geneva: WHO; 1996.

4. World Health Organization (WHO). Making a difference in countries: strategic approach to improving maternal and newborn survival and health: ensuring skilled care for every birth. Geneva: WHO; 2006.

5. Kinney MV, Kerber KJ, Black RE, Cohen B, Nkrumah F, Coovadia H, et al. Sub-Saharan Africa's mothers, newborns, and children: where and why do they die? PLoS Med. 2010;7:e1000294.

6. Hogan MC, Foreman KJ, Naghavi M, Ahn SY, Wang M, Makela SM, et al. Maternal mortality for 181 countries, 1980-2008: a systematic analysis of progress towards Millennium Development Goal 5. Lancet. 2010;375:1609-23.

7. Rajaratnam JK, Marcus JR, Flaxman AD, Wang H, Levin-Rector A, Dwyer L, et al. Neonatal, postneonatal, childhood, and under-5 mortality for 187 countries, 1970-2010: a systematic analysis of progress towards Millennium Development Goal 4. Lancet. 2010;375:1988-2008. 
8. United Nations Development Programme (UNDP). Beyond the midpoint: achieving the Millennium Development Goals. New York: UNDP; 2010

9. Martines J, Paul VK, Bhutta ZA, Koblinsky M, Soucat A, Walker N, et al. Neonatal survival: a call for action. Lancet. 2005:365:1189-97.

10. Lawn JE, Tinker A, Munjanja SP, Cousens S. Where is maternal and child health now? Lancet. 2006;368:1474-7.

11. Kerber KJ, de Graft-Johnson JE, Bhutta ZA, Okong P, Starrs A, Lawn JE. Continuum of care for maternal, newborn, and child health: from slogan to service delivery. Lancet. 2007;370:1358-69.

12. McBryde-Foster M, Allen T. The continuum of care: a concept development study. J Adv Nurs. 2005;50:624-32.

13. McKelvey RS. A continuum of mental health care for children and adolescents. Hosp Community Psychiatry. 1988;39:870-3.

14. Fujita M, Poudel KC, Do TN, Bui DD, Nguyen VK, Green K, et al. A new analytical framework of 'continuum of prevention and care' to maximize HIV case detection and retention in care in Vietnam. BMC Health Serv Res. 2012; 12:483.

15. The Partnership for Maternal, Newborn and Child Health (PMNCH). Strategic Framework 2012 to 2015. Geneva: World Health Organization; 2011.

16. Tinker A, ten Hoope-Bender P, Azfar S, Bustreo F, Bell R. A continuum of care to save newborn lives. Lancet. 2005:365:822-5.

17. World Health Organization (WHO). The World Health Report 2005: make every mother and child count. Geneva: WHO; 2005.

18. Lassi ZS, Majeed A, Rashid S, Yakoob MY, Bhutta ZA. The interconnections between maternal and newborn health-evidence and implications for policy. J Matern Fetal Neonatal Med. 2013;26 Suppl 1:3-53.

19. Jokhio $\mathrm{AH}$, Winter HR, Cheng KK. An intervention involving traditional birth attendants and perinatal and maternal mortality in Pakistan. N Engl J Med. 2005;352:2091-9.

20. Manandhar DS, Osrin D, Shrestha BP, Mesko N, Morrison J, Tumbahangphe $\mathrm{KM}$, et al. Effect of a participatory intervention with women's groups on birth outcomes in Nepal: cluster-randomised controlled trial. Lancet. 2004;364:970-9.

21. McPherson RA, Tamang J, Hodgins S, Pathak LR, Silwal RC, Baqui AH, et al. Process evaluation of a community-based intervention promoting multiple maternal and neonatal care practices in rural Nepal. BMC Pregnancy Childbirth. 2010;10:31.

22. Bhutta ZA, Memon ZA, Soofi S, Salat MS, Cousens S, Martines J. Implementing community-based perinatal care: results from a pilot study in rural Pakistan. Bull World Health Organ. 2008;86:452-9.

23. Kumar V, Mohanty S, Kumar A, Misra RP, Santosham M, Awasthi S, et al. Effect of community-based behaviour change management on neonatal mortality in Shivgarh, Uttar Pradesh, India: a cluster-randomised controlled trial. Lancet. 2008;372:1151-62.

24. Gamble C, Ekwaru PJ, Garner P, ter Kuile FO. Insecticide-treated nets for the prevention of malaria in pregnancy: a systematic review of randomised controlled trials. PLoS Med. 2007:4:e107.

25. Scholl TO, Hediger ML, Fischer RL, Shearer JW. Anemia vs iron deficiency: increased risk of preterm delivery in a prospective study. Am J Clin Nutr. 1992;55:985-8.

26. Garn SM, Ridella SA, Petzold AS, Falkner F. Maternal hematologic levels and pregnancy outcomes. Semin Perinatol. 1981;5:155-62.

27. Bahl R, Qazi S, Darmstadt GL, Martines J. Why is continuum of care from home to health facilities essential to improve perinatal survival? Semin Perinatol. 2010;34:477-85.

28. Witter S, Adjei S, Armar-Klemesu M, Graham W. Providing free maternal health care: ten lessons from an evaluation of the national delivery exemption policy in Ghana. Glob Health Action. 2009;2. doi: 10.3402/gha.v2i0.1881

29. Basinga P, Gertler PJ, Binagwaho A, Soucat AL, Sturdy J, Vermeersch CM. Effect on maternal and child health services in Rwanda of payment to primary health-care providers for performance: an impact evaluation. Lancet. 2011:377:1421-8.

30. Lim SS, Dandona L, Hoisington JA, James SL, Hogan MC, Gakidou E. India's Janani Suraksha Yojana, a conditional cash transfer programme to increase births in health facilities: an impact evaluation. Lancet. 2010;375:2009-23.

31. The Partnership for Maternal, Newborn and Child Health (PMNCH). Opportunities for Africa's newborns: practical data, policy and programmatic support for newborn care in Africa. Geneva: World Health Organization; 2006.

32. de Graft-Johnson JDP, Otchere S, Russell N, Bell R. Household-to-home continuum of maternal and newborn care. Maryland: ACCESS Programme; 2005.
33. Sibley LM, Sipe TA, Brown CM, Diallo MM, McNatt K, Habarta N. Traditiona birth attendant training for improving health behaviours and pregnancy outcomes. Cochrane Database Syst Rev. 2007;3:CD005460.

34. Hussein J, Kanguru L, Astin M, Munjanja S. The effectiveness of emergency obstetric referral interventions in developing country settings: a systematic review. PLoS Med. 2012;9:e1001264.

35. Abato MHJ. Conditional cash transfer programs and health in conditional cash transfers in Latin America. Maryland: Jophns Hopkins University Press; 2010.

36. David HP, Taghreed A, Olakunle A, Irene Akua A, Nhan T. Implementation research: what it is and how to do it. BMJ. 2013;347:f6753.

37. Darmstadt GL, Bhutta ZA, Cousens S, Adam T, Walker N, de Bernis L. Evidence-based, cost-effective interventions: how many newborn babies can we save? Lancet. 2005;365:977-88

38. Darmstadt GL, Walker N, Lawn JE, Bhutta ZA, Haws RA, Cousens S. Saving newborn lives in Asia and Africa: cost and impact of phased scale-up of interventions within the continuum of care. Health Policy Plann. 2008;23:101-17.

39. Naoto Kan PMJ. Promise to the next generation. The high-level plenary meeting of the sixty-fifth session of the general assembly of the United Nations; 2010. http://www.un.org/en/mdg/summit2010/debate/JP_en.pdf. Accessed 4 Jan 2015

40. Ministry of Foreign Affairs of Japan. Japan's Global Health Policy: 2011-2015. Tokyo: Ministry of Foreign Affairs of Japan; 2010.

41. Okada K. Japan's new global health policy: 2011-2015. Lancet. 2010;376:938-40

42. Takahashi K, Kobayashi J, Nomura-Baba M, Kakimoto K, Nakamura Y. Can Japan contribute to the Post Millennium Development Goals? Making human security mainstream through the TICAD process. Trop Med Health. 2013:41:135-42

43. Sugiyama HYA, Murakami H. Japan's Global Health Policy; developing a comprehensive approach in period of economic stress. Lanham: Rowman \& Littlefield Publishers; 2013.

44. Ghana Statistical Service (GSS) Ghana Health Service, ICF Macro. Ghana Maternal Health Survey 2007. Maryland: ICF Macro; 2008.

45. Ghana Statistical Service (GSS) Ghana Health Service, ICF Macro. Ghana Demographic and Health Survey. Maryland: ICF Macro; 2009

46. World Health Organization (WHO). World Health Statistics 2011. Geneva: WHO; 2011.

47. Adjuik M, Kanyomse E, Kondayire F, Wak G, Hodgson A. Clustering of under-five mortality in the Navrongo HDSS in the Kassena-Nankana District of northern Ghana. Glob Health Action. 2010;3. doi: 10.3402/gha.v3i0.5233

48. Mills S, Williams JE, Wak G, Hodgson A. Maternal mortality decline in the Kassena-Nankana district of northern Ghana. Matern Child Health J. 2008:12:577-85.

49. Nettey OE, Zandoh C, Sulemana A, Adda R, Owusu-Agyei S. Clustering of childhood mortality in the Kintampo Health and Demographic Surveillance System in Ghana. Glob Health Action. 2010;3. doi: 10.3402/gha.v3i0.5258

50. Awini E, Mattah P, Sankoh O, Gyapong M. Spatial variations in childhood mortalities at the Dodowa Health and Demographic Surveillance System site of the INDEPTH Network in Ghana. Trop Med Int Health. 2010;15:520-8.

51. Engmann C, Walega P, Aborigo RA, Adongo P, Moyer CA, Lavasani L, et al. Stillbirths and early neonatal mortality in rural northern Ghana. Trop Med Int Health. 2012;17:272-82.

52. Welaga $\mathrm{P}$, Moyer CA, Aborigo R, Adongo P, Williams J, Hodgson A, et al. Why are babies dying in the first month after birth? A 7-year study of neonatal mortality in northern Ghana. PLoS One. 2013;8:e58924.

53. World Health Organization (WHO). Countdown to 2015: taking stock of maternal, newborn and child survival. Decade Report (2000-2010). Geneva: WHO; 2010.

54. Bazzano AN, Kirkwood BR, Tawiah-Agyemang C, Owusu-Agyei S, Adongo PB. Beyond symptom recognition: care-seeking for ill newborns in rural Ghana. Trop Med Int Health. 2008;13:123-8.

55. Oiyemhonlan B, Udofia E, Punguyire D. Identifying obstetrical emergencies at Kintampo Municipal Hospital: a perspective from pregnant women and nursing midwives. Afr J Reprod Health. 2013;17:129-40.

56. Kayode GA, Ansah E, Agyepong IA, Amoakoh-Coleman M, Grobbee DE, Klipstein-Grobusch K. Individual and community determinants of neonatal mortality in Ghana: a multilevel analysis. BMC Pregnancy Childbirth. 2014;14:165.

57. Moran AC, Kerber K, Sitrin D, Guenther T, Morrissey CS, Newby H, et al. Measuring coverage in $\mathrm{MNCH}$ : indicators for global tracking of newborn care. PLoS Med. 2013;10:e1001415. 
58. Okyere E, Tawiah-Agyemang C, Manu A, Deganus S, Kirkwood B, Hill Z. Newborn care: the effect of a traditional illness, asram, in Ghana. Ann Trop Paediatr. 2010;30:321-8.

59. Victora CG, Hanson K, Bryce J, Vaughan JP. Achieving universal coverage with health interventions. Lancet. 2004;364:1541-8.

60. Haws RA, Thomas AL, Bhutta ZA, Darmstadt GL. Impact of packaged interventions on neonatal health: a review of the evidence. Health Policy Plann. 2007:22:193-215.

61. Curran GM, Bauer M, Mittman B, Pyne JM, Stetler C. Effectivenessimplementation hybrid designs: combining elements of clinical effectiveness and implementation research to enhance public health impact. Med Care. 2012;50:217-26.

62. Zwarenstein M, Treweek S, Gagnier JJ, Altman DG, Tunis S, Haynes B, et al. Improving the reporting of pragmatic trials: an extension of the CONSORT statement. BMJ. 2008:337:a2390.

63. Rogers E, Fernandez S, Gillespie C, Smelson D, Hagedorn HJ, Elbel B, et al. Telephone care coordination for smokers in VA mental health clinics: protocol for a hybrid type-2 effectiveness-implementation trial. Addict Sci Clin Pract. 2013:8:7.

64. Brownson RCCG, Proctor EK. Dissemination and implementation research in health: translating science to practice. Oxford: Oxford University Press; 2012.

65. INDEPTH Network. Accra, Ghana. http://www.indepth-network.org (1998) Accessed 4 Jan 2015

66. Gyapong M, Sarpong D, Awini E, Manyeh AK, Tei D, Odonkor G, et al. Profile: the Dodowa HDSS. Int J Epidemiol. 2013:42:1686-96.

67. Owusu-Agyei S, Nettey OE, Zandoh C, Sulemana A, Adda R, Amenga-Etego $S$, et al. Demographic patterns and trends in Central Ghana: baseline indicators from the Kintampo Health and Demographic Surveillance System. Glob Health Action. 2012;5:1-11.

68. Bazzano AN, Kirkwood B, Tawiah-Agyemang C, Owusu-Agyei S, Adongo P. Social costs of skilled attendance at birth in rural Ghana. Int J Gynaecol Obstet. 2008:102:91-4.

69. Oduro AR, Wak G, Azongo D, Debpuur C, Wontuo P, Kondayire F, et al Profile of the Navrongo Health and Demographic Surveillance System. Int J Epidemiol. 2012;41:968-76

70. Macleod B, Phillips J, Stone AE, Walji A, Awoonor-Williams JK. The architecture of a software system for supporting community-based primary health care with mobile technology: the mobile technology for community health (MoTeCH) initiative in Ghana. Online J Public Health Inform. 2012;4. doi:10.5210/ojphi.v4i1.3910

71. Duysburgh E, Williams A, Williams J, Loukanova S, Temmerman M. Quality of antenatal and childbirth care in northern Ghana. BJOG. 2014;121 Suppl 4:117-26.

72. Awoonor-Williams JK, Bawah AA, Nyonator FK, Asuru R, Oduro A, Ofosu A, et al. The Ghana essential health interventions program: a plausibility trial of the impact of health systems strengthening on maternal \& child survival. BMC Health Serv Res. 2013;13 Suppl 2:S3.

73. Group NSA, Bahl R, Bhandari N, Dube B, Edmond K, Fawzi W, et al. Efficacy of early neonatal vitamin A supplementation in reducing mortality during infancy in Ghana, India and Tanzania: study protocol for a randomized controlled trial. Trials. 2012;13:22.

74. Kirkwood BR, Manu A, Tawiah-Agyemang C, ten Asbroek G, Gyan T, Weobong $B$, et al. NEWHINTS cluster randomised trial to evaluate the impact on neonatal mortality in rural Ghana of routine home visits to provide a package of essential newborn care interventions in the third trimester of pregnancy and the first week of life: trial protocol. Trials. 2010;11:58.

75. Ghana-Luxembourg Social Trust. International Labour Organization, Switzerland. http://www.lo.org/addisababa/WCMS_304015/lang-en/index. htm (2009). Accessed 4 Jan 2015.

76. World Health Organization (WHO). Consultation on definitions and standards related to maternal and child health and the perinatal period. WHO. 1987. http://www.who.int/iris/handle/10665/61544\#sthash.HFPDKIKk. dpuf. Accessed 4 Jan 2015.

77. World Health Organization (WHO). International statistical classification of diseases and related health problems 10th revision. WHO. 1995. http://apps. who.int/classifications/icd10/browse/2015/en. Accessed 4 Jan 2015.
78. Proctor E, Silmere H, Raghavan R, Hovmand P, Aarons G, Bunger A, et al. Outcomes for implementation research: conceptual distinctions, measurement challenges, and research agenda. Adm Policy Ment Health. 2011;38:65-76.

79. Rabin BA, Brownson RC, Haire-Joshu D, Kreuter MW, Weaver NL. A glossary for dissemination and implementation research in health. J Public Health Manag Pract. 2008;14:117-23.

80. Eldridge SKS. A practical guide to cluster randomised trials in health services research. New York: John Wiley \& Sons; 2012.

\section{Submit your next manuscript to BioMed Central and take full advantage of:}

- Convenient online submission

- Thorough peer review

- No space constraints or color figure charges

- Immediate publication on acceptance

- Inclusion in PubMed, CAS, Scopus and Google Scholar

- Research which is freely available for redistribution 